Pacific Journal of Mathematics

ISOMORPHIC POWER SERIES RINGS 


\title{
ISOMORPHIC POWER SERIES RINGS
}

\author{
M. J. O'MALley
}

Let $A$ and $B$ be commutative rings with identity, let $X$ be an indeterminate over $A$ and $B$, and let $A[[X]]$ and $B[[X]]$ be the formal power series rings over $A$ and $B$, respectively.

The motivation for this paper was to consider the analogue of a question raised by Coleman and Enochs for the polynomial ring. Specifically, the following question is considered:

$$
\text { (*) If } \quad A[[X]] \cong B[[X]] \text {, must } A \cong B \text { ? }
$$

(The author knows no counterexample.)

The paper is divided into three parts. The first part, $\S 2$, deals with the problem of extending a given homomorphism $\rho$ of $A$ into $B[[X]]$ to a homomorphism $\phi$ of $A[[X]]$ into $B[[X]]$ such that $\phi$ maps $X$ onto an arbitrary element $\beta$ of $B[[X]]$. Necessary and sufficient conditions are given in order that there exists an extension of $\rho$ to an isomorphism $\phi$ of $A[[X]]$ onto $B[[X]]$ such that $\phi(X)=\beta$. In $\S 3$, it is shown that if $\phi$ is an isomorphism of $A[[X]]$ onto $B[[X]]$ such that either $\phi(A) \subseteq B$ or $B \subseteq \phi(A)$, then $\phi(A)=B$, so that $\phi$ itself is an isomorphism of $A$ onto $B$. In $\S 4$, sufficient conditions are imposed on $A$ to guarantee that the condition $A[[X]] \cong B[[X]]$ implies that $A \cong B$. In particular, if $A$ is semisimple (the Jacobson radical of $A=(0))$, then the condition $A[[X]] \cong B[[X]]$ implies that $A \cong B$.

All rings considered in this paper are assumed to be commutative and contain an identity element. The symbols $\omega$ and $\omega_{0}$ are used throughout the paper to denote the sets of positive and nonnegative integers, respectively. If $g=\sum_{i=0}^{\infty} c_{i} X^{i}$ is a nonzero element of $B[[X]]$ such that the first nonzero coefficient of $g$ is $c_{k}$, then we say that $g$ has order $k$ and we write $O(g)=k$.

Throughout the paper, our notation will be the same as that of [4]. In particular, if $\beta \in B[[X]]$ and if $T$ is a subring of $B[[X]]$ containing $\beta$ and the identity of $B$, then $\left(\beta^{n} T\right)$ will denote the ideal of $T$ generated by $\beta^{n}$, and $(T,(\beta T))$ the topological ring $T$ in the $(\beta T)$-adic topology. It is well known that $(T,(\beta T))$ is a Hausdorff space if and only if $\bigcap_{n \in \omega}\left(\beta^{n} T\right)=(0)$. [6; p. 252]. We say that $(T,(\beta T))$ is complete if each Cauchy sequence of $T$ converges to a point of $T$. If $U$ is a subring of $B[[X]]$, then $U[\beta]$ will be the subring of $B[[X]]$ consisting of all finite sums of the form: $\sum_{i=0}^{n} u_{i} \beta^{i}, u_{i} \in U$.

We denote the Jacobson radical of a ring $A$ by $J(A)$ and we make frequent use of the two facts: 
p. 206].

(i) $J(A)=\{a \in A \mid 1-r a$ is a unit of $A$ for all $r \in A\}$. [5;

(ii ) $J(A[[X]])=J(A) A[[X]]+(X) . \quad[3 ;$ p. 62].

2. Homomorphisms of power series rings. Let $\beta \in B[[X]]$ and suppose that $\rho$ is a homomorphism of $A$ into $B[[X]]$. In this section we consider the problem of extending $\rho$ to a homomorphism $\phi$ of $A[[X]]$ into $B[[X]]$ such that $\phi(X)=\beta$. O'Malley [3] and O'Malley and Wood [4] have considered this problem for the case when $A=B$ and $\rho$ is the identity map. Several, but not all, of the results of [4], together with their proofs, extend to the general case with only minor modifications. We summarize the results of interest here and reference the corresponding result in [4]. Throughout this section, we suppose that $\rho$ maps the identity of $A$ onto the identity of $B$, and since there will be no ambiguity, we write 1 to denote either identity. Note that if an extension $\phi$ of $\rho$ exists for which $B \subseteq \phi(A[[X]])$, then the condition $\rho(1)=1$ is necessarily satisfied. Throughout this section, we denote $\rho(A)$ by $U$.

We first observe that if $T$ is a subring of $B[[X]]$ containing $U[\beta]$ and such that $(T,(\beta T))$ is a complete Hausdorff space, then we may define a mapping $\phi$ from $A[[X]]$ into $B[[X]]$ by $\phi(f)=\lim _{n} \sum_{i=0}^{n} \rho\left(f_{i}\right) \beta^{i}$, for each $f=\sum_{i=0}^{\infty} f_{i} X^{i} \in A[[X]]$, where the limit is taken in $(T,(\beta T))$. Following the proof for Theorem (2.2) given in [4], it can easily be shown that $\phi$, as defined above, is the unique homomorphism of $A[[X]]$ into $B[[X]]$ that extends $\rho$, maps $X$ onto $\beta$, and has range contained in $T$.

We note that if $\bigcap_{n \in \omega}\left(\beta^{n}\right)=(0)$ (in $\left.B[[X]]\right)$, and if $\phi$ is a homomorphism of $A[[X]]$ into $B[[X]]$ such that $\phi / A=\rho$ and $\phi(X)=\beta$, then it can be shown that $\phi$ is the unique homomorphism of $A[[X]]$ into $B[[X]]$ with these two properties. In particular, if $\phi$ is an isomorphism (onto $B[[X]]$ ), then $\bigcap_{n \in \omega}\left(\beta^{n}\right)=\bigcap_{n \in \omega}(\phi(X))^{n}=\phi\left(\bigcap_{n \in \omega}\left(X^{n}\right)\right)=$ $\phi((0))=(0)$, and hence, $\phi$ is the unique homomorphism of $A[[X]]$ into $B[[X]]$ that extends $\rho$ and maps $X$ onto $\beta$.

We now observe that if we impose the added condition on $T$ that each element of $T$ be the limit of a Cauchy sequence of (U[B], $(\beta U[\beta]))$, then we have the following theorem.

THEOREM 2.1. Let $T$ be a subring of $B[[X]]$ containing $U[\beta]$ and satisfying each of the following:

(i) $(T,(\beta T))$ is a complete Hausdorff space;

(ii) Each element of $T$ is the limit of a Cauchy sequence of $(U[\beta],(\beta U[\beta]))$.

Then there exists a unique homomorphism $\phi$ of $A[[X]]$ into $B[[X]]$ 
that extends $\rho$, maps $X$ onto $\beta$, and has range equal to $T$.

Conversely, let $\phi$ be a homomorphism of $A[[X]]$ into $B[[X]]$ such that $\phi / A=\rho$ and $\phi(X)=\beta$. If $T=\phi(A[[X]])$, and if $\bigcap_{n \in \omega}\left(\beta^{n} T\right)=(0)$, then $T$ is a subring of $B[[X]]$ containing $U[\beta]$ and satisfying conditions (i) and (ii).

\section{Proof. See [4; Theorem (2.3)].}

For the one-to-one case, we have the following characterization. We note that this is the first result that does not carry over intact from [4]. Part (iii) of Example (2.6) will provide an example of an isomorphism $\phi$ of $A[[X]]$ onto $B[[X]]$ such that $\phi(X)=\beta=\sum_{i=0}^{\infty} b_{i} X^{i}$ and such that $\beta-b_{0}=0$. In particular, $\beta-b_{0}$ is not regular in $U[\beta]$, and hence the change here in condition (i) of Theorem (2.2) from that of condition (i) of Theorem (3.2) in [4] is necessary.

TheOREM 2.2. Let $\beta=\sum_{i=0}^{\infty} b_{i} X^{i} \in B[[X]]$ and suppose that $\rho$ is a homomorphism of $A$ into $B[[X]]$. There exists a one-to-one homomorphism $\phi$ of $A[[X]]$ into $B[[X]]$ such that $\phi(X)=\beta$ and $\phi / A=\rho$ if and only if the following conditions are satisfied:

(i) $\beta$ is transcendental over $U$.

(ii) $\rho$ is one-to-one.

(iii) There exists a subring $T$ of $B[[X]]$ such that $(T,(\beta T))$ is a completion of $(U[\beta], \beta U[\beta])$.

Proof. [4; Lemma (3.1) and Theorem (3.2)].

Observe that by Theorem 2.1, if $\bigcap_{n \in \omega}\left(\beta^{n}\right)=(0)$ (in $B[[X]]$ ), then there exists a homomorphism $\phi$ of $A[[X]]$ onto $B[[X]]$ such that $\rho(X)=\beta$ and $\phi / A=\rho$ if and only if $(B[[X]]),(\beta))$ is complete and $(U[\beta],(\beta U[\beta]))$ is dense in $(B[[X]],(\beta))$. Thus with Theorem 2.2 and its proof, we have the following.

THEOREM 2.3. Under the hypothesis of Theorem 2.2, there exists an isomorphism $\phi$ of $A[[X]]$ onto $B[[X]]$ such that $\phi(X)=\beta$ and $\phi / A=\rho$ if and only if the following conditions are satisfied:

(i) $\beta$ is transcendental over $U$.

(ii) $\rho$ is one-to-one.

(iii) $(B[[X]],(\beta))$ is a completion of $(U[\beta],(\beta U[\beta]))$. Moreover, when such an isomorphism $\phi$ exists, $\phi$ is the unique isomorphism of $A[[X]]$ onto $B[[X]]$ that extends $\rho$ and maps $X$ onto $\beta$.

REMARK 2.4. It is clear from the preceding results of this section that the conditions obtained here to guarantee the existence 
of an extension of $\rho$ to a homomorphism (isomorphism) $\phi$ of $A[[X]]$ into (onto) $B[[X]]$ such that $\phi(X)=\beta$ are very similar to the conditions obtained in [4] to guarantee the existence of a $B$-endomorphism (automorphism) $\psi$ of $B[[X]]$ such that $\psi(X)=\beta$. In particular, if $\phi$ is an extension of $\rho$ to an isomorphism of $A[[X]]$ onto $B[[X]]$ such that $\phi(X)=\beta$, then $(B[[X]],(\beta))$ is a complete Hausdorff space (Theorem 2.3), and hence, by [4; Theorem 2.2], there exists a $B$-endomorphism $\psi$ of $B[[X]]$ such that $\psi(X)=\beta$. A natural question then is: must $\psi$ be an automorphism of $B[[X]]$ ? The answer is no in general, but we are able to obtain necessary and sufficient conditions in order that the answer be yes. We provide these conditions (under slightly more general hypothesis) in Theorem 2.5, and in part (iii) of Example 2.6 we provide an example of rings $A$ and $B$ and an isomorphism $\phi$ of $A[[X]]$ onto $B[[X]]$ for which the corresponding $B$-endomorphism of $B[[X]]$ is neither one-to-one nor onto.

However, before doing this, we first observe that if $A[[X]] \cong$ $B[[X]]$ under $\phi$, and if the corresponding $B$-endomorphism $\psi$ of $B[[X]]$ such that $\psi(X)=\phi(X)$ is, in fact, an automorphism, then we have an affirmative answer to our question $(*)$. For, in this case, if $\tau=\psi^{-1} \circ \phi$, then $\tau$ is an isomorphism of $A[[X]]$ onto $B[[X]]$ such that $\tau(X)=X$. Hence,

$$
A \cong A[[X]] /(X) \cong B[[X]] /(\tau(X))=B[[X]] /(X) \cong B
$$

This observation is a useful tool in seeking to obtain sufficient conditions on $A$ to guarantee an affirmative answer to $\left(^{*}\right)$. It was used extensively in [1] in considering the analogous question for the polynomial ring, and we use it in $\S 4$ of this paper.

THEOREM 2.5. Let $\beta \in B[[X]]$ be such that $\bigcap_{n \in \omega}\left(\beta^{n}\right)=(0)$ and let $\phi$ be a homomorphism of $A[[X]]$ onto $B[[X]]$ such that $\phi(X)=\beta$. Then the unique B-endomorphism $\psi$ of $B[[X]]$ such that $\psi(X)=\beta$ is an automorphism if and only if $U=\phi(A) \subseteq \psi(B[[X]])$.

Proof. First note that since $\phi$ is onto and since $\bigcap_{n \in \omega}\left(\beta^{n}\right)=(0)$, then by the comment preceding Theorem $2.3(B[[X]],(\beta))$ is a complete Hausdorff space and $(U[\beta],(\beta U[\beta]))$ is dense in $(B[[X]],(\beta))$. In particular, since $(B[[X]],(\beta))$ is a complete Hausdorff space, there exists a unique $B$-endomorphism $\psi$ of $B[[X]]$ such that $\psi(X)=\beta$.

If $\psi$ is an automorphism, then clearly $\phi(A) \subseteq \psi(B[[X]])=B[[X]]$. Conversely, if $U=\phi(A) \subseteq \psi(B[[X]])$, then, since $\beta \in \psi(B[[X]])$, we have that $U[\beta] \subseteq \psi(B[[X]])$. Thus, since $(U[\beta],(\beta U[\beta]))$ is dense in $(B[[X]],(\beta))$, it follows that $(\psi(B[[X]]),(\beta \psi(B[[X]])))$ is dense in $(B[[X]],(\beta))$. But $(\psi(B[[X]]),(\beta \psi(B[[X]])))$ is a complete Hausdorff 
space $([4$; Theorem 2.3]), and thus it follows that $\psi(B[[X]])=B[[X]]$. Therefore, $\psi$ is onto and hence is an automorphism of $B[[X]]$. [4; Corollary 4.6].

One of the main results of [4; Theorem 4.7] shows that if $\psi$ is a $B$-endomorphism of $B[[X]]$ such that $\psi(X)=\sum_{i=0}^{\infty} b_{i} X^{i}=\beta$, then the following conditions are equivalent.

(a) $\psi$ is onto.

(b) $\psi$ is an automorphism.

(c) $b_{1}$ is a unit of $B$.

The only implication true for the general case is the obvious one $(b) \rightarrow(a)$. We provide counterexamples to show that none of the other implications are true. It suffices to show that (c) $\nrightarrow$ (a), (a) $\nrightarrow(\mathrm{b})$, and (b) $\rightarrow$ (c). Note that for each of our examples $A=B$.

EXAMPLE 2.6. Let $R$ be a ring, let $\left\{Y_{i}\right\}_{i \in \omega}$ be an infinite collection of indeterminates over $R$, and let $A=B=R\left[\left\{Y_{i}\right\}_{i \in \omega}\right]$ be the polynomial ring in an infinite number of indeterminates over $R$. Let $X$ be an indeterminate over $B$; we use $B[[X]]$ and $\beta=X$ for the first two examples.

(i ) (c) $\nrightarrow$ (a). Define $\rho: B \rightarrow B[[X]]$ by $\rho(r)=r$, for each $r \in R$, and $\rho\left(Y_{i}\right)=Y_{i+1}$ for each $i \in \omega$. Then $\rho$ determines a unique one-toone homomorphism of $B$ into $B[[X]]$, and, since $(B[[X]],(X))$ is a complete Hausdorff space, we may extend $\rho$ to an endomorphism $\phi$ of $B[[X]]$ such that $\phi(X)=X=\beta$. Since $Y_{1} \notin$ range of $\phi, \phi$ is not onto, but $b_{1}=1$ is a unit of $B$.

(ii) (a) $\nrightarrow$ (b). Define $\rho: B \rightarrow B[X]]$ by $\rho(r)=r$, for each $r \in R$, $\rho\left(Y_{i+1}\right)=Y_{i}$ for each $i \in \omega$, and $\rho\left(Y_{1}\right)=X$. Then the unique one-toone homomorphism of $B$ into $B[[X]]$ determined by $\rho$ has range $=$ $B[X]$. Since $B[X][X]=B[X]$ and since $(B[[X]],(X))$ is the completion of $(B[X],(X B[X]))$, there exists an extension of $\rho$ to an endomorphism $\phi$ of $B[[X]]$ onto $B[[X]]$ such that $\phi(X)=X$. Clearly, $\phi$ is not one-to-one.

(iii) (b) $\nrightarrow$ (c). Let $R$ be a ring, $X, Y$ indeterminates over $R$, and $R[[X, Y]]=R[[Y]][[X]]$ the formal power series ring in $X$ and $Y$. If $A=B=R[[Y]]$, then the mapping $\phi$ from $B[[X]]$ into itself that is the identity on $R$ and interchanges $X$ and $Y$ is clearly an automorphism of $B[[X]]$ and, in particular, $\phi(X)=\sum_{i=0}^{\infty} b_{i} X^{i}$, where $b_{0}=Y$ and $b_{i}=0$, for each $i \geqq 1$.

3. Isomorphisms of $A[[X]]$ onto $B[[X]]$ that are isomorphisms of $A$ onto $B$. In [1], it is shown that if $\sigma: A[X] \rightarrow B[X]$ is an isomorphism such that $\sigma(A) \subseteq B$, then $\sigma(A)=B$. As an easy consequence of this result is the fact that the same conclusion holds, if the reverse containment is assumed. The main purpose of this 
section will be to prove the corresponding results for the power series ring.

TheOREM 3.1. Let $\beta=\sum_{i=0}^{\infty} b_{i} X^{i} \in B[[X]]$ and suppose that $\phi$ is an isomorphism of $A[[X]]$ onto $B[[X]]$ such that $\phi(X)=\beta$. If $\phi(A) \subseteq B$, then $\phi(A)=B$.

We require a few preliminary observations before proving Theorem 3.1. We make strong use of the results of [4]. We begin with a lemma.

LEMMA 3.2. Let $\sigma: A[[X]] \rightarrow B[[X]]$ be a homomorphism such that $\sigma(X)=\gamma=\sum_{i=0}^{\infty} c_{i} X^{i}$. If $B \subseteq \sigma(A[[X]])$, then $c_{0} \in J(B)$. In particular, if $\sigma$ is onto, then $c_{0} \in J(B)$.

Proof. For each $g \in A[[X]], 1+g X$ is a unit of $A[[X]]$, and hence, $\sigma(1+g X)=1+\sigma(g) \gamma$ is a unit of $\sigma(A[[X]])$. Thus, if $B \subseteq$ $\sigma(A[[X]])$, then, for each $b \in B$, there exists a $g \in A[[X]]$ such that $\sigma(g)=b$, and hence $\sigma(1+g X)=1+b \gamma=\left(1+b c_{0}\right)+\sum_{i=1}^{\infty} b c_{i} X^{i}$ is a unit of $B[[X]]$. Therefore, by [6; p. 131], $1+b c_{0}$ is a unit of $B$ for each $b \in B$, and thus, $c_{0} \in J(B)$. [5; p. 206].

Suppose that $\phi$ is an isomorphism of $A[[X]]$ onto $B[[X]]$ satisfying the hypothesis of Theorem 3.1. If $\psi$ denotes the unique $B$ endomorphism of $B[[X]]$ that maps $X$ onto $\beta$, then $\phi(A) \cong B \subseteq$ $\psi(B[[X]])$, and hence, by Theorem 2.5, $\psi$ is an automorphism. Therefore, $b_{1}$ is a unit of $B$. [4; Theorem 4.5].

Of prime importance to us is the following.

THEOREM 3.3. Under the hypothesis of Theorem 3.1, there exists an A-automorphism $\tau$ of $A[[X]]$ such that $\tau(X)=\phi^{-1}\left(-b_{0}\right)+X$.

Proof. Let $\phi(h)=-b_{0} ; h=\sum_{i=0}^{\infty} h_{i} X^{i} \in A[[X]]$. Then $\phi(h+X)=$ $\beta-b_{0}$ and $\phi^{-1}\left(\beta-b_{0}\right)=h+X$. Thus, since $\phi^{-1}:\left(B[[X]],\left(\beta-b_{0}\right)\right) \rightarrow$ $(A[[X]],(h+X))$ is continuous, and since $\left(B[[X]],\left(\beta-b_{0}\right)\right)$ is a complete Hausdorff space, it follows that $(A[[X]],(h+X))$ is a complete Hausdorff space. Therefore, by [4; Theorem 2.2], there exists a unique $A$-endomorphism $\tau$ of $A[[X]]$ such that $\tau(X)=h+X=$ $\phi^{-1}\left(\beta-b_{0}\right)=\phi^{-1}\left(-b_{0}\right)+X$. We show that $\tau$ is an automorphism.

Consider $\phi(h)=\phi\left(\sum_{i=0}^{\infty} h_{i} X^{i}\right)=\lim _{n} \sum_{i=0}^{n} \phi\left(h_{i}\right) \beta^{i}=-b_{0}$, where the limit is taken in the $(\beta)$-adic topology on $B[[X]]$. It follows that, for $k \geqq 2$, there exists $N_{k} \in \omega$ such that $\sum_{i=0}^{n} \phi\left(h_{i}\right) \beta^{i}+b_{0} \in(\beta)^{k}$ for $n \geqq N_{k}$. Thus, if $\sum_{i=0}^{n} \phi\left(h_{i}\right) \beta^{i}+b_{0}=v \beta^{k}$, then, expanding both sides of the equality in powers of $X$, we have that the coefficient of $X$ on the left-hand side has the form $\phi\left(h_{1}\right) b_{1}+\sum_{i=1}^{n-1} \phi\left(h_{i+1}\right) c_{i} b_{0}^{i}$, where $c_{i} \in B$ 
for each $i$, while the coefficient of $X$ on the right-hand side belongs to $\left(b_{0}\right)$. Therefore, since $b_{0} \in J(B)$ (Lemma 3.2), and since $b_{1}$ is a unit of $B$, it follows that $\phi\left(h_{1}\right) \in J(B)$, and hence $h_{1} \in J(A)$. Thus, $1+h_{1}$, the coefficient of $X$ in $\tau(X)$, is a unit of $A$, so that $\tau$ is an $A$-automorphism of $A[[X]]$. [4; Theorem 4.7].

Proof of Theorem 3.1. Consider the isomorphism $\mu=\phi \circ \tau$ of $A[[X]]$ onto $B[[X]]$. Since $\phi \circ \tau(X)=\phi\left(\phi^{-1}\left(-b_{0}\right)+X\right)=\beta-b_{0}$, and since $\phi \circ \tau(\alpha)=\phi(a)$ for each $a \in A$, it follows from the definition and comments preceding Theorem 2.1 that, for each $g=\sum_{i=0}^{\infty} g_{i} X^{i} \in A[[X]]$, $\mu(g)=\lim _{n} \sum_{i=0}^{n} \phi\left(g_{i}\right)\left(\beta-b_{0}\right)^{i}=\sum_{i=0}^{\infty} \phi\left(g_{i}\right)\left(\beta-b_{0}\right)^{i}$. Thus, since $\mu$ is onto, for each $b \in B$, there exists $g=\sum_{i=0}^{\infty} g_{i} X^{i} \in A[[X]]$ such that $\mu(g)=\sum_{i=0}^{\infty} \phi\left(g_{i}\right)\left(\beta-b_{0}\right)^{i}=b$. But since $O\left(\beta-b_{0}\right)=1$ and since $\phi(A) \subseteq$ $B$, it follows that $\phi\left(g_{0}\right)=b$. Therefore, $B \cong \phi(A)$, and thus we have equality.

COROLLARY 3.4. Let $\beta=\sum_{i=0}^{\infty} b_{i} X^{i} \in B[[X]]$ and suppose that $\phi$ is an isomorphism of $A[[X]]$ onto $B[[X]]$ such that $\phi(X)=\beta$. If $B \subseteq$ $\phi(A)$, then $\phi(A)=B$.

Proof. Since $B \subseteq \phi(A), \phi^{-1}(B) \subseteq A$. Thus, $\phi^{-1}$ is an isomorphism of $B[[X]]$ onto $A[[X]]$ such that $\phi^{-1}(B) \subseteq A$. Therefore, by Theorem 3.1, $\phi^{-1}(B)=A$, and hence, $B=\phi\left(\phi^{-1}(B)\right)=\phi(A)$.

In connection with Theorem 3.1 and Corollary 3.4 it should be remarked here that if $\rho: A \rightarrow B$ is an isomorphism and if $\beta \in B[[X]]$, then the existence of an extension of $\rho$ to a homomorphism $\phi$ of $A[[X]]$ into $B[[X]]$ such that $\phi(X)=\beta$ is equivalent to the existence of a $B$-endomorphism $\psi$ of $B[[X]]$ such that $\psi(X)=\beta$. It can be shown that this correspondence establishes a one-to-one relationship between the set of homomorphisms of $A[[X]]$ into $B[[X]]$ which restrict to $\rho$ on $A$ and the set of $B$-endomorphisms of $B[[X]]$.

In particular, we have the following theorem. We note that it is the analogue of a result of Gilmer for the polynomial ring. [2; Theorem 4].

THEOREM 3.5. Let $\rho$ be an isomorphism of $A$ onto $B$ and let $\beta \in B[[X]]$. There exists an extension of $\rho$ to a homomorphism $\phi$ of $A[[X]]$ into $B[[X]]$ such that $\phi(X)=\beta$ if and only if there exists a $B$-endomorphism is of $B[[X]]$ such that $\psi(X)=\beta$. Moreover, if such an extension $\phi$ exists, then:

(i ) $\phi$ is the unique extension of $\rho$ to a homomorphism of $A[[X]]$ into $B[[X]]$ that maps $X$ onto $\beta$ if and only if $\psi$ is the unique $B$-endomorphism of $B[[X]]$ that maps $X$ onto $\beta$. 
(ii) $\phi$ is one-to-one if and only if $\psi$ is one-to-one.

(iii) $\phi$ is onto if and only if $\psi$ is onto. Hence, by (ii) and (iii), $\phi$ is an isomorphism if and only if $\psi$ is a B-automorphism of $B[[X]]$.

Proof. Observe that if such an extension $\phi$ exists, then $\psi: B[[X]] \rightarrow B[[X]]$ defined by $\psi\left(\sum_{i=0}^{\infty} f_{i} X^{i}\right)=\phi\left(\sum_{i=0}^{\infty} \rho^{-1}\left(f_{i}\right) X^{i}\right)$ is a $B$ endomorphism of $B[[X]]$ such that $\psi(X)=\beta$. Conversely, if such a $B$-endomorphism $\psi$ exists, then $\phi: A[[X]] \rightarrow B[[X]]$ defined by $\phi\left(\sum_{i=0}^{\infty} a_{i} X^{i}\right)=\psi\left(\sum_{i=0}^{\infty} \rho\left(a_{i}\right) X^{i}\right)$ is an extension of $\rho$ to a homomorphism of $A[[X]]$ into $B[[X]]$ such that $\phi(X)=\beta$.

The verification of these statements, together with (i), (ii), and (iii), is straightforward, and we omit it.

4. Strongly power invariant rings. In this section, in contrast to $\S 3$, we attempt to impose conditions on $A$ (rather than $\phi$ ) to guarantee that the condition $A[[X]] \cong B[[X]]$ implies that $A \cong B$.

Extending the terminology used in [1], we say that a ring $A$ is power invariant if whenever $B$ is a ring such that $A[[X]]$ is isomorphic to $B[[X]]$, then $A$ and $B$ are isomorphic. A ring $A$ is said to be strongly power invariant if whenever $B$ is a ring and $\phi$ is an isomorphism of $A[[X]]$ onto $B[[X]]$, then there exists a $B$-automorphism $\psi$ of $B[[X]]$ such that $\psi(X)=\phi(X)$. We have already implicitly observed in Remark 2.4 that a strongly power invariant ring is power invariant. However, part (iii) of Example 2.6 shows that $R[[X]]$ is not strongly power invariant for any ring $R$.

In this section we shall be concerned only with the property: strongly power invariant. Our main result is that any semisimple ring $A(J(A)=(0))$ is strongly power invariant.

Observe that if $R$ and $S$ are rings and if $\phi$ is a homomorphism of $R$ onto $S$, then $\phi(J(R)) \subseteq J(S)$, and if, in addition, the kernel of $\phi$ is contained in $J(R)$, equality holds.

We have a simple, but key lemma.

LEMMA 4.1. Let $\beta=\sum_{i=0}^{\infty} b_{i} X^{i} \in B[[X]]$ and suppose that $\phi$ is $a$ homomorphism of $A[[X]]$ onto $B[[X]]$ such that $\phi(X)=\beta$. If the kernel of $\phi$ is contained in $J(A[[X]])=J(A)+(X)$, and if $\phi(J(A)) \subseteq$ $B$, then $b_{1}$ is a unit of $B$.

Proof. By the previous remark, $\phi(J(A)+(X))=\phi(J(A))+(\beta)=$ $J(B)+(X)$. Thus, there exists $g \in \phi(J(A)), \quad h=\sum_{i=0}^{\infty} h_{i} X^{i} \in B[[X]]$ such that $X=g+h \beta$. Since $\phi(J(A)) \subseteq B, g \in B$, and therefore, equating coefficients of $X$, we have that $1=h_{0} b_{1}+h_{1} b_{0}$. By Lemma 
$3.2, b_{0} \in J(B)$, and thus $h_{0} b_{1}=1-h_{1} b_{0}$ is a unit of $B$, so that $b_{1}$ is a unit of $B$.

Lemma 4.2. Under the hypothesis of Lemma 4.1, if $\phi$ is an isomorphism, then there exists a B-automorphism \& of $B[[X]]$ such that $\psi(X)=\beta$.

Proof. We have already observed (Remark 2.4) that if $\phi$ is an isomorphism then there exists a $B$-endomorphism $\psi$ of $B[[X]]$ such that $\psi(X)=\beta$. Hence, by Lemma $4.1, b_{1}$ is a unit of $B$, and thus $\psi$ is an automorphism. [4; Theorem 4.7].

RESUlt 4.3. For any ring $A, A / J(A)$ is strongly power invariant. In particular, if $A$ is a semisimple ring, then $A$ is strongly power invariant.

Proof. Let $C=A / J(A)$ and let $B$ be a ring having the property that there exists an isomorphism $\phi$ of $C[[X]]$ onto $B[[X]]$. If $\phi(X)=\beta=\sum_{i=0}^{\infty} b_{i} X^{i}$ and if $\psi$ is the corresponding $B$-endomorphism of $B[[X]]$ mapping $X$ onto $\beta$, then, since $J(C)=(0)$, Lemma (4.2) implies that $b_{1}$ is a unit of $B$, and therefore, $\psi$ is an automorphism.

Observing that the units of $B[X]$ coincide with the units of $B$ if and only if $B$ has no nonzero nilpotent element, we have the following.

CoRollary 4.4. Let $\beta=\sum_{i=0}^{\infty} b_{i} X^{2} \in B[[X]]$ and suppose that $\phi$ is an isomorphism of $A[[X]]$ onto $B[[X]]$ such that $\dot{\phi}(X)=\beta$. If $A$ has no nonzero nilpotent element and if $\phi(A) \cong B[X]$, then $A \cong B$.

Proof. Since $A$ has no nonzero nilpotent element, it follows that $A[[X]]$ has no nonzero nilpotent element. Thus, since $\phi$ is an isomorphism, we have that $B[[X]]$, and in particular, $B$, has no nonzero nilpotent element. Thus, if $t \in J(A)$, then $1-t$ is a unit of $A$, and hence, $\phi(1-t)=1-\phi(t)$ is a unit of $\phi(A) \leqq B[X]$. By the preceding observation, it follows that $1-\phi(t) \in B$, so that $\phi(t) \in B$. Thus, $\phi(J(A)) \subseteq B$, and hence, it follows from Lemma 4.2 that there exists a $B$-automorphism $\psi$ of $B[[X]]$ such that $\psi(X)=\beta$. Therefore, as observed in Remark 2.4, $A \cong B$.

\section{REFERENCES}

1. D. Coleman and E. Enochs, Isomorphic polynomial rings, Proc. Amer. Math. Soc., 27 (1971), 247-252.

2. R. W. Gilmer, R-automorphisms of $R[X]$, Proc. London Math. Soc., (3) 18 (1968), 328-336. 
3. M. O'Malley, R-automorphisms of $R[[X]]$, Proc. London Math. Soc., (3) 20 (1970), 60-78.

4. M. O'Malley and C. Wood, R-endomorphisms of $R[[X]]$, J. Algebra, 15 (1970), $314-327$.

5. O. Zariski and P. Samuel, Commutative Algebra, I, Van Nostrand, Princeton, 1958. 6. - Commutative Algebra II, Van Nostrand, Princeton, 1960.

Received January 28, 1971 and in revised form August 12, 1971.

Nasa, Manned Spacecraft Center, Houston, Texas 


\section{PACIFIC JOURNAL OF MATHEMATICS}

\section{EDITORS}

\author{
H. SAMELSON \\ Stanford University \\ Stanford, California 94305

\section{R. HoвBY} \\ University of Washington \\ Seattle, Washington 98105
}

\section{J. DugundJI}

Department of Mathematics University of Southern California Los Angeles, California 90007

\author{
RichaRd ARENS \\ University of California \\ Los Angeles, California 90024
}

\section{ASSOCIATE EDITORS}
E. F. BECKENBACH
B. H. NEUMANN
F. WOLF
K. YoshidA

\section{SUPPORTING INSTITUTIONS}

\author{
UNIVERSITY OF BRITISH COLUMBIA \\ CALIFORNIA INSTITUTE OF TECHNOLOGY \\ UNIVERSITY OF CALIFORNIA \\ MONTANA STATE UNIVERSITY \\ UNIVERSITY OF NEVADA \\ NEW MEXICO STATE UNIVERSITY \\ OREGON STATE UNIVERSITY \\ UNIVERSITY OF OREGON \\ OSAKA UNIVERSITY
}

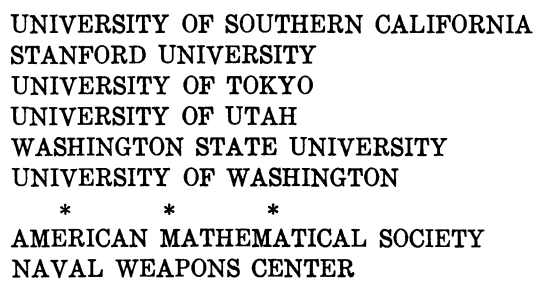

The Supporting Institutions listed above contribute to the cost of publication of this Journal, but they are not owners or publishers and have no responsibility for its content or policies.

Mathematical papers intended for publication in the Pacific Journal of Mathematics should be in typed form or offset-reproduced, (not dittoed), double spaced with large margins. Underline Greek letters in red, German in green, and script in blue. The first paragraph or two must be capable of being used separately as a synopsis of the entire paper. The editorial "we" must not be used in the synopsis, and items of the bibliography should not be cited there unless absolutely necessary, in which case they must be identified by author and Journal, rather than by item number. Manuscripts, in dup icate if possible, may be sent to any one of the four editors. Please classify according to the scheme of Math. Rev. Index to Vol. 39. All other communications to the editors should be addressed to the managing editor, Richard Arens, University of California, Los Angeles, California, 90024.

50 reprints are provided free for each article; additional copies may be obtained at cost in multiples of 50 .

The Pacific Journal of Mathematics is published monthly. Effective with Volume 16 the price per volume (3 numbers) is $\$ 8.00$; single issues, $\$ 3.00$. Special price for current issues to individual faculty members of supporting institutions and to individual members of the American Mathematical Society: $\$ 4.00$ per volume; single issues $\$ 1.50$. Back numbers are available.

Subscriptions, orders for back numbers, and changes of address should be sent to Pacific Journal of Mathematics, 103 Highland Boulevard, Berkeley, California, 94708.

PUBLISHED BY PACIFIC JOURNAL OF MATHEMATICS, A NON-PROFIT CORPORATION

Printed at Kokusai Bunken Insatsusha (International Academic Printing Co., Ltd.), 270, 3-chome Totsuka-cho, Shinjuku-ku, Tokyo 160, Japan. 


\section{Pacific Journal of Mathematics}

\section{Vol. 41, No. 2 December, 1972}

Tom M. (Mike) Apostol, Arithmetical properties of generalized Ramanujan sums .......................................... 281

David Lee Armacost and William Louis Armacost, On p-thetic groups ........ 295

Janet E. Mills, Regular semigroups which are extensions of groups .......... 303

Gregory Frank Bachelis, Homomorphisms of Banach algebras with minimal ideals ................................................ 307

John Allen Beachy, A generalization of injectivity .................. 313

David Geoffrey Cantor, On arithmetic properties of the Taylor series of rational functions. II.........................................

Václáv Chvátal and Frank Harary, Generalized Ramsey theory for graphs. III.

Small off-diagonal numbers .................................. 335

Frank Rimi DeMeyer, Irreducible characters and solvability of finite groups . . . . 347

Robert P. Dickinson, On right zero unions of commutative semigroups........ 355

John Dustin Donald, Non-openness and non-equidimensionality in algebraic

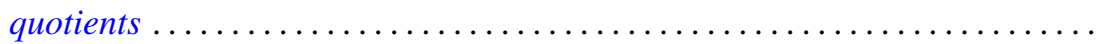

John D. Donaldson and Qazi Ibadur Rahman, Inequalities for polynomials with a prescribed zero ........................................ 375

Robert E. Hall, The translational hull of an $N$-semigroup ................ 379

John P. Holmes, Differentiable power-associative groupoids.............. 391

Steven Kenyon Ingram, Continuous dependence on parameters and boundary data for nonlinear two-point boundary value problems .

Robert Clarke James, Super-reflexive spaces with bases ..........

Gary Douglas Jones, The embedding of homeomorphisms of the plane in

continuous flows...............................

Mary Joel Jordan, Period $H$-semigroups and $t$-semisimple periodic

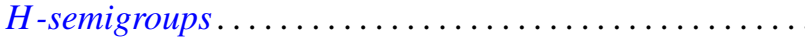

Ronald Allen Knight, Dynamical systems of characteristic 0

Kwangil Koh, On a representation of a strongly harmonic ring by sheaves...

Hui-Hsiung Kuo, Stochastic integrals in abstract Wiener space. ..

Thomas Graham McLaughlin, Supersimple sets and the problem of extending a

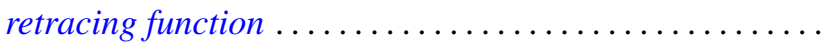

William Nathan, Open mappings on 2-manifolds .

M. J. O'Malley, Isomorphic power series rings

Sean B. O'Reilly, Completely adequate neighborhood systems and metrization

Qazi Ibadur Rahman, On the zeros of a polynomial and its derivative...

Russell Daniel Rupp, Jr., The Weierstrass excess function ..

Hugo Teufel, A note on second order differential inequalities and functional

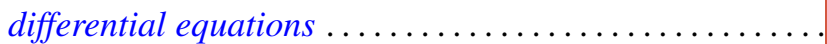

M. J. Wicks, A general solution of binary homogeneous equations over free 